\title{
Langerhans Cell Histiocytosis Presenting as a Unifocal Eosinophilic Granuloma in the Body of the Mandible: A Rare Report
}

\author{
${ }^{1}$ Pratik Dipak Shah, ${ }^{2}$ Srijon Mukherji
}

\begin{abstract}
Introduction: Langerhans cell histiocytosis $(\mathrm{LCH})$ is a relatively rare and unique benign disease characterized by an abnormal proliferation of immature dendritic cells which usually affects children and young adults. Jaws are involved in less than $10 \%$ of children with the disease.
\end{abstract}

Objective: To add on to the literature one more case of this rare and unique disease which accounts for less than $1 \%$ of all bone tumors and also to discuss its oral manifestation and management.

Design: Case report

Setting: Private maxillofacial surgery institute, Kolkata, West Bengal.

Patient: A 2 years and 8 months old male child with solitary swelling over the lower left jaw since one and half months. Lesion was fixed to underlying bone and there was an expansion of buccal cortical plate at the body of mandible.

Results: Enucleation of the lesion performed after assessing history, clinical and radiological dertails and also considering patient's age. Postoperative biopsy result came as $\mathrm{LCH}$ presenting as a unifocal eosinophil granuloma. Appropriate surgical intervention resulted in a dramatic improvement in patient's clinical condition. At present, patient is disease-free and asymptomatic.

Conclusion: Surgical enucleation or curettage is a preferred treatment option for maxillary and mandibular lesion. Potential for the unifocal disease to become multifocal should not be underestimated as the disease can be unpredictable. We recommend long-term follow-up due to uncertainty of the disease.

Keywords: Langerhans cell histiocytosis, Eosinophilic granuloma, Benign.

\section{Source of support: Nil}

Conflict of interest: None

\footnotetext{
${ }^{1}$ Surgical Fellow, ${ }^{2}$ Director and Chief Consultant

${ }^{1,2}$ Department of Oral and Maxillofacial Surgery, Calcutta Institute of Maxillofacial Surgery, Kolkata, West Bengal, India
}

Corresponding Author: Pratik Dipak Shah, Surgical Fellow Department of Oral and Maxillofacial Surgery, Calcutta Institute of Maxillofacial Surgery, Kolkata, West Bengal, India, Phone: 03324412366, e-mail: pratikdshah.2711@gmail.com

\section{INTRODUCTION}

Langerhans cell histiocytosis (LCH) is a rare and unique disorder of the reticuloendothelial system characterized by an abnormal proliferation of histiocytes and eosinophilic leukocytes. ${ }^{1}$ Lichtenstein gave the term histiocytosis $\mathrm{X}$ in 1953 to include three clinical varieties including eosinophilic granuloma, Hand-Schüller-Christian, and Letterer-Siwe disease, which shared some common histologic features and clinical findings. ${ }^{2}$ The origin of the disease was believed to be inflammatory rather than neoplastic. ${ }^{3}$ Clinical manifestations may range from single or multifocal bone lesions to disseminated oral disease with multiorgan involvement. Incidence rate for eosinophilic granuloma of bone ranges from 1 new case in 350000 to 2 million per year which is an uncommon disease of maxillofacial region. ${ }^{4}$ Histiocytosis $\mathrm{X}$ localized (EG) refers to a form of the disease typified by a solitary or a few multiple skeletal terms without extraskeletal extension; it usually affects children and young adults. Eosinophilic granuloma may not present physical signs or symptoms in the clinical observation and most of the times it is discovered during routine radiographic examination. Apart from skull bones and mandible, other bones like femur, ribs, humerus may also be affected.

\section{CASE REPORT}

A two years and eight months old male child was referred to Calcutta Institute of Maxillofacial Surgery and Research Institute in the month of October 2013 for the complain of solitary painless swelling of left side of the lower jaw since one and half months (Fig. 1). Swelling was insidious in onset which started as a small mass initially and gradually progressed to present size. Clinical examination revealed extraoral swelling over the left side of the mandible. Intraorally lesion was seen at the body of the mandible. Lesion was around $2.5 \times 2.5 \mathrm{~cm}$ in diameter, bony hard, immovable and nontender in nature. There was expansion of buccal cortical plate over left side of the body of the mandible. Skin over the swelling was smooth and pinchable and did not show any secondary changes. There was no functional deficit of inferior alveolar nerve in the area of distribution. Orthopantamogram revealed a well-defined 


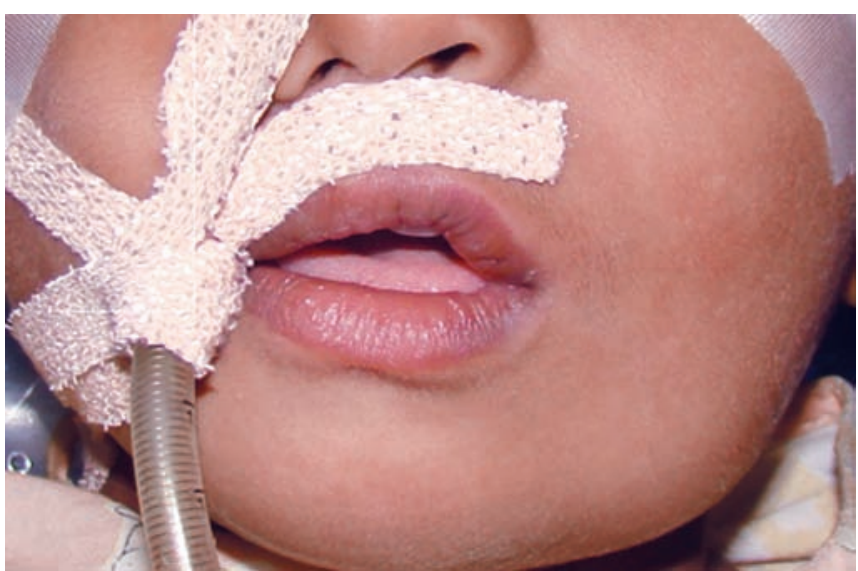

Fig. 1: Swelling over the left side of the body of the mandible

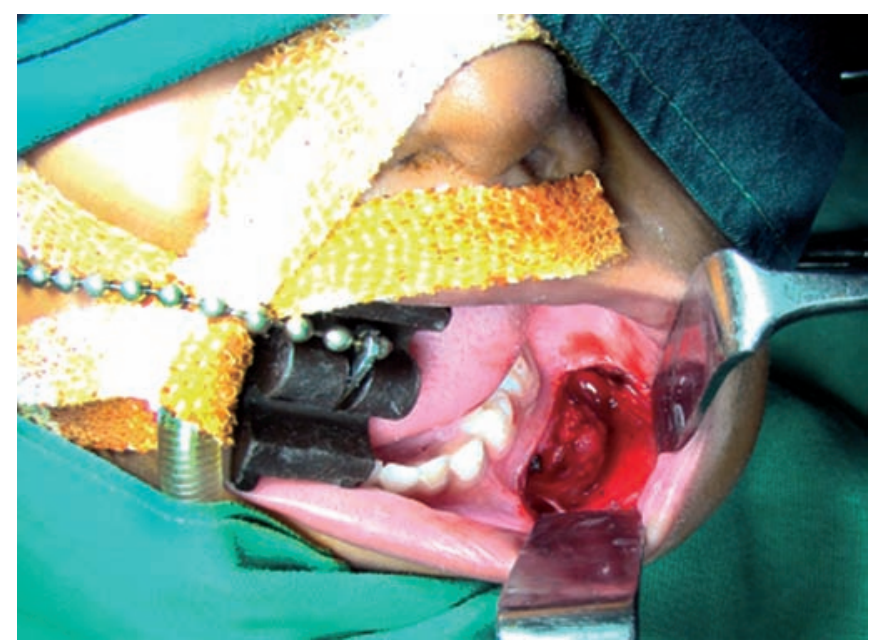

Fig. 3: Lesion identified and exposed through vestibular incision

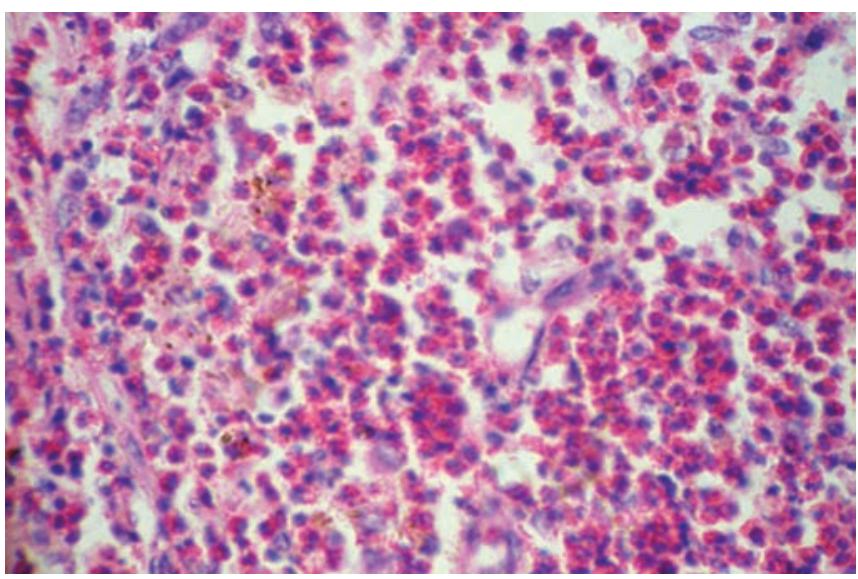

Fig. 5: Histopathology showing profuse infiltration of eosinophils, plasma cells, lymphocytes and extravasated RBC in connective tissue stroma

punched out lesion with reactive sclerosis between the roots of 75 (Fig. 2). Patient was undertaken for enucleation of the lesion under general anesthesia. Aspiration of the lesion was performed with wide bore needle to rule out vascular component. Two centimeter length incision was placed in gingivobuccal sulcus of left side of mandibular body region.

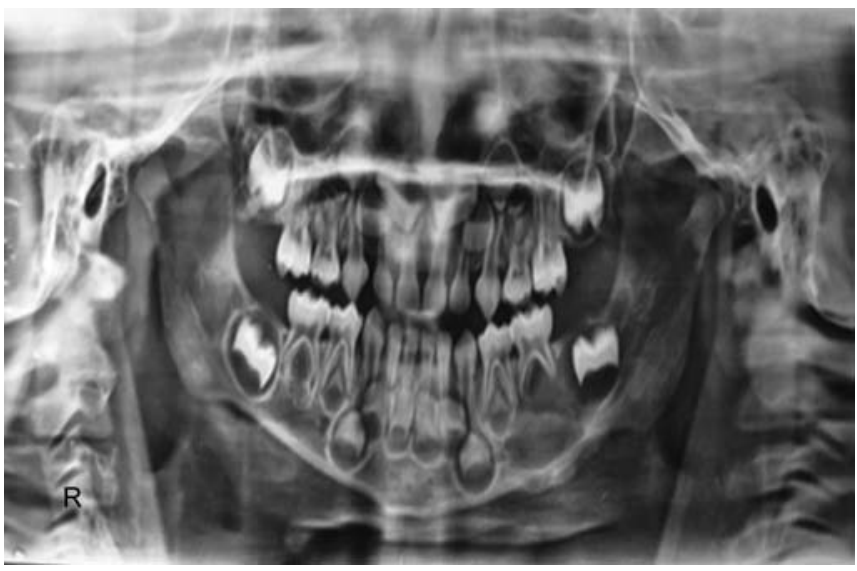

Fig. 2: OPG showing osteolytic lesion periapical to 75

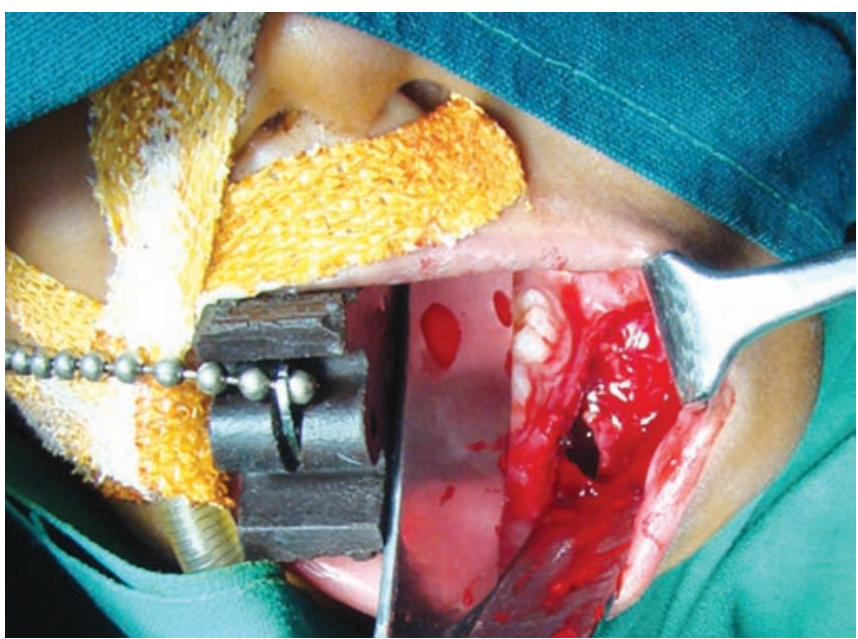

Fig. 4: Surgical defect after enucleation of the lesion

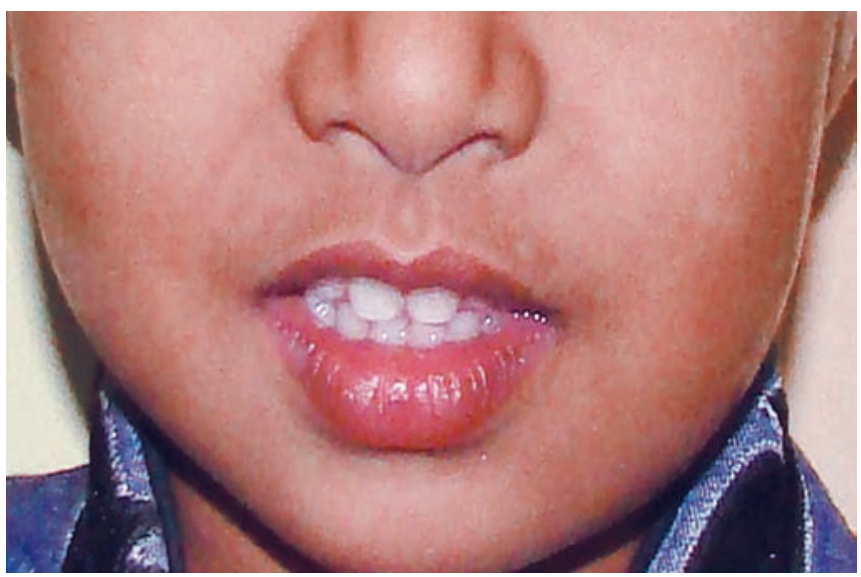

Fig. 6: Three months postoperatively

Subperiosteal flap was reflected. Lesion was identified and exposed (Fig. 3). Enucleation of the lesion was performed followed by surgical removal of 75 (Fig. 4). Hemostasis was achieved and closure was performed with vicryl 4-0. Histopathological examination revealed areas of histiocytic proliferation, granulomatous tissue with profuse infiltration 
of eosinophils, plasma cells, lymphocytes and extravasated RBC in highly cellular and vascular connective tissue stroma (Fig. 5). There was a presence of Langerhan's cells and multinucleated giant cells in fibrocollagenous tissue which were suggestive of unifocal periapical eosinophilic granuloma. Immunohistochemical staining with CD1a and S-100 protein confirmed the diagnosis. Patient is being followed up at regular intervals (Fig. 6).

\section{DISCUSSION}

In $90 \%$ of the reported cases, it appears in children under the age of ten with peak incidence between one and four years of age. The lesion may occur in the jaw and overlying soft tissue of the mouth. Reported incidence rate of the disease involving maxilla and mandible is $7.9 \% .^{5}$ There is a certain predilection to males in the ratio of $2: 1 .^{6}$ Unifocal eosinophilic granuloma may present with swelling, tenderness, loss of superficial alveolar bone and localized periodontitis and premature exfoliation of teeth. It has bone destructive nature and it is well-demarcated, roughly round or oval in shape. The area which is destroyed is replaced by soft tissue and later the lesion becomes fibrous. ${ }^{7}$ In the present case site of occurrence, age, sex, nature of the disease were compatible with those written in previous reports. The etiology and pathogenesis remains unclear. Varieties of etiological factors have been proposed including immunologic reactions, viruses, bacteria and genetic influences. ${ }^{8,9}$ Possible development of LCH under the influence of colony stimulating factor (GM-CSF), interleukin-3, and tumor necrosis factor-alpha have also been suggested and recently, cytogenic studies have proposed the role of tumor suppressor genes (p53), oncogenes (c-myc, h-ras), growth factors, cell surface immunologic markers and apoptotic factors in $\mathrm{LCH}$ as well. ${ }^{10}$ Localized, unifocal and severe osteolytic nature are the characteristics of unifocal eosinophilic granuloma, which may be easily mistaken for either severe localized periodontitis or periapical infection. These osseous lesions may be wrongly diagnosed as prepubertal periodontitis in children. Osteomyelitis, one of the possible diagnoses, was ruled out in the present case as the history regarding trauma to the jaws and tooth infection was negative. A wide spectrum of treatment modalities have so far been adopted, ranging from surgical curettage to radio and/or chemotherapy. ${ }^{11}$ Accessible lesions are best managed by surgical excision or curettage, since it promotes healing in most of the cases. In multifocal disease variants associated with dysfunction of vital organs, immunosuppressive treatments have been suggested with recently introduced 2- chloro-2- deoxyadenosine (2-CdA) (Cladribine). However, the type and extent of the disease responding to this therapy needs further clarification.

\section{CONCLUSION}

Advances in immune-histochemistry, molecular biology, and radiodiagnostic techniques may help in proper understanding of this variable group of disorders. Potential for the unifocal disease to become multifocal should not be underestimated as the disease can be unpredictable. It is important to include eosinophilic granuloma in the differential diagnosis of bone lesions in adult subjects because of the possible expansion of the disease if untreated. There is general agreement that surgical curettage is the preferred treatment for maxillary and mandibular lesions and that radiotherapy and chemotherapy should be reserved for lesions that are not surgically manageable. We recommend a long-term follow-up due to unpredictable nature of the disease.

\section{REFERENCES}

1. Moghadam BK, Saedi S, Gier RE. Adult-onset multifocal histiocytosis X presenting as a periodontal problem. J Oral Maxillofac Surg 1991Apr;49(4):417-419.

2. Lichtenstein L. Histiocytosis X: integration of eosinophilic granuloma of bone, Letterer-Siwe disease and Schüller-Christian disease as related manifestations of a single nosologic entity. AMA Arch Pathol 1953 Jul;56(1):84-102.

3. Glick D, et al. Histocytosis X: report of two cases. Oral Health 1979;69:35-38.

4. Agarwal A, Agrawal GP, Alam S, Husain B. A case of unifocal eosinophilic Granuloma of the mandible in an adult female: a case report. Case Reports in Dentis. Volume 2012, Article ID 521726.

5. Gunashekhar M, Ponnudurai A. Langerhans cell histiocytosis involving maxilla and mandible. J Dent, Tehran University of Medical Sciences 2009;6(2).

6. Ando A, Hatori M, Hosaka M, Hagiwara Y, Kita A, Itoi E. Eosinophilic granuloma arising from the pelvis in children: a report of three cases. Upsala J Med Sci 2008;113(2):209-215.

7. Terro W. Histiocytosis-X: unifocal eosinophilic granuloma. A case report with 8 years follow-up. Dental News 1997;4(3).

8. Lombardi T, Hauser C, Budtz-Jörgensen E. Langerhans cells: structure, function and role in oral pathological conditions. J Oral Pathol Med 1993 May;22(5):193-202.

9. Hanapiah F, Yaacob H, Ghani KS, Hussin AS. Histiocytosis X: evidence for a genetic etiology. J Nihon Univ Sch Dent 1993 Sep;35(3):171-174.

10. Murakami I, Gogusev J, Fournet JC, Glorion C, Jaubert F. Detection of molecular cytogenetic aberrations in langerhans cell histiocytosis of bone. Hum Pathol 2002 May;33(5):555-560.

11. Can IH, Kurt A, Ozer E, Sari N, Samim E. Mandibular manifestation of Langerhans cell histiocytosis in children. Oral Oncol Extra 2005 Sep;41(8):174-177. 